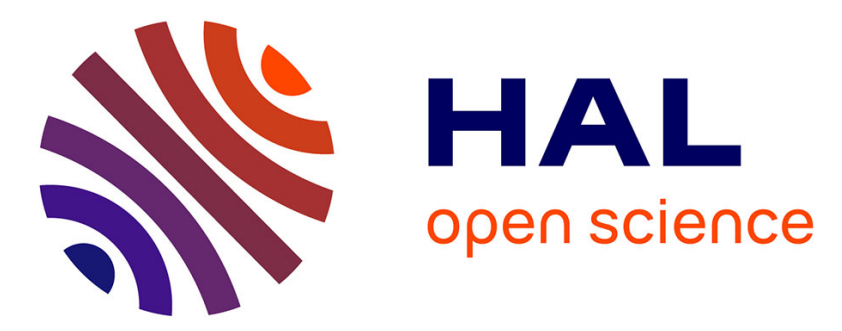

\title{
A Biophysical Model of Shape Changes due to Atrophy in the Brain with Alzheimer's Disease
}

\author{
Bishesh Khanal, Marco Lorenzi, Nicholas Ayache, Xavier Pennec
}

\section{To cite this version:}

Bishesh Khanal, Marco Lorenzi, Nicholas Ayache, Xavier Pennec. A Biophysical Model of Shape Changes due to Atrophy in the Brain with Alzheimer's Disease. MICCAI 2014 - 17th International Conference Medical Image Computing and Computer-Assisted Intervention, Sep 2014, Boston, United States. pp.41-48, 10.1007/978-3-319-10470-6_6 . hal-01006478

\section{HAL Id: hal-01006478 \\ https://inria.hal.science/hal-01006478}

Submitted on 16 Jun 2014

HAL is a multi-disciplinary open access archive for the deposit and dissemination of scientific research documents, whether they are published or not. The documents may come from teaching and research institutions in France or abroad, or from public or private research centers.
L'archive ouverte pluridisciplinaire HAL, est destinée au dépôt et à la diffusion de documents scientifiques de niveau recherche, publiés ou non, émanant des établissements d'enseignement et de recherche français ou étrangers, des laboratoires publics ou privés. 


\title{
A Biophysical Model of Shape Changes due to Atrophy in the Brain with Alzheimer's Disease
}

\author{
Bishesh Khanal, Marco Lorenzi, Nicholas Ayache, and Xavier Pennec \\ INRIA Sophia Antipolis Méditerranée, Asclepios Research Project \\ 2004 Route des Lucioles - BP 93, 06902 Sophia Antipolis, France \\ \{bishesh.khanal, marco.lorenzi, nicholas.ayache, xavier.pennec\}@inria.fr
}

\begin{abstract}
This paper proposes a model of brain deformation triggered by atrophy in Alzheimer's Disease (AD). We introduce a macroscopic biophysical model assuming that the density of the brain remains constant, hence its volume shrinks when neurons die in $\mathrm{AD}$. The deformation in the brain parenchyma minimizes the elastic strain energy with the prescribed local volume loss. The cerebrospinal fluid (CSF) is modelled differently to allow for fluid readjustments occuring at a much faster time-scale. PDEs describing the model is discretized in staggered grid and solved using Finite Difference Method. We illustrate the power of the model by showing different deformation patterns obtained for the same global atrophy but prescribed in gray matter (GM) or white matter (WM) on a generic atlas MRI, and with a realistic $\mathrm{AD}$ simulation on a subject MRI. This well-grounded forward model opens a way to study different hypotheses about the distribution of brain atrophy, and to study its impact on the observed changes in MR images.
\end{abstract}

Keywords: Alzheimer's disease, Biophysical model, Atrophy model, Atrophy Simulation, Longitudinal modeling

\section{Introduction}

$\mathrm{AD}$ is characterized by volume loss in the brain [3]. For this reason, various segmentation and registration methods in brain MR imaging have been proposed to model and quantify volume changes in AD [6]. These methods can however provide different results depending on the assumptions they are based on. The estimation of brain atrophy from longitudinal images is an inverse problem since volume changes have to be estimated from the observed data. Registration and segmentation methods implicitly assume a backward model whose parameters must be inferred. These parameters are usually difficult to relate to the underlying biophysical process. For instance, non linear registration results depend on the regularization imposed on the transformation [1], and can therefore lead to different atrophy patterns for the same observed follow-up MRIs. One would wonder how sensitive such measurements are to the diverse registration or segmentation assumptions, and what is the related impact on the understanding of the dynamics of brain atrophy due to $\mathrm{AD}$. This is an important issue for clinical applications of image based computational methods in AD. 
A different approach to the problem is to define a suitable forward biophysical model of anatomical changes that can simulate plausible atrophy patterns. By controlling the defined parameters, the model should ideally provide simulations that can be easily related to the biophysical assumptions. This model would represent a valuable reference for testing clinical hypotheses on the pathological evolution, and at the same time, can be used for the benchmarking of atrophy measurement methods.

Atrophy simulators [9][12][15][5] have been proposed and used mostly for the validation of registration or segmentation methods [4][13], or to estimate uncertainty in the measured atrophy [14]. We can broadly distinguish two major approaches used in such simulators: Jacobian based, and bio-mechanical based.

In Jacobian based methods [9][12][13], the desired level of atrophy is set at each voxel, and the deformation that best approximates the desired level of atrophy is found. Regularization is used in the optimization to enforce certain desired conditions such as topology preservation. The advantage of these methods is the ability to define atrophy maps at the voxel level. However regularization parameters used to enforce topology preservation are generally difficult to relate to a plausible biophysical process of $\mathrm{AD}$. Moreover, it is not trivial to consider different tissue behaviors.

Biomechanical models generate tissue deformation based on biomechanical principles. As far as we know, the only model proposed so far for AD application is a thermoelastic one [15][5]. The authors defined the volume changes in particular structures and tissues of a meshed brain by assigning different thermal coefficients. Simulation of the images is done by first solving the thermoelastic model of tissue deformatiom with Finite Element Method (FEM), and then by interpolating the obtained displacement field from the mesh to the image. The main problem of this type of approach is once again the difficulty to relate thermal coefficients to a physical model of neuronal death. Moreover, FEM involves moving back and forth from voxels to meshes which creates numerical difficulties and inaccuracies in the model personalization.

In this work we combine the best of both approaches by proposing a biophysically plausible atrophy model based on the prescribed atrophy rate at each voxel of the parenchyma. The model results in brain deformations which strictly satisfy the constraints imposed by the prescribed atrophy rate. In the following section we explain the assumptions and the mathematical formulation of the proposed model. Section 3 provides the implementation details and the experimental setup. Simulation results for a generic atlas template and a subject specific patient are shown in section 4.

\section{Model of Deformation of the Brain with Atrophy}

Brain could be seen as a deformable material that floats in the CSF enclosed by a rigid skull. Atrophy results in the shrinkage and in the structural readjustment of the brain tissues. The first important point to notice is that we cannot directly apply the classical continuum mechanics formulation because conservation of 
mass does not hold due to the presence of atrophy. Secondly, the CSF volume increases to compensate the tissue volume loss and the skull does not deform. However, the CSF production is at a much smaller time-scale (hours) compared to the atrophy (months). To take into account these observations, we model the CSF and the brain parenchyma differently while fixing the skull as the boundary condition.

\subsection{Loss of Volume and Conservation Equation}

It is well known that neurons die as AD progresses. In diseases like CreutzfeldtJakob disease, no gross brain shape changes are reported and the imaging only shows hyperintense signals on T2-weighted images [8]. However, this is not the case in AD and longitudinal MRIs show a significant decrease of brain volume instead [6]. We assume that after the death of cells, remodeling of the tissue occurs such that the density remains constant. In other words, abstracting the events of AD to macroscopic level, we assume that the volume loss corresponds to the reduction of the brain mass. This assumption of incompressible material but with mass loss leads us to the conservation law given by: $\nabla \cdot \boldsymbol{v}=-\tilde{a}$, where $\boldsymbol{v}$ is a velocity field corresponding to the deformation of the brain and $\tilde{a}$ is the atrophy rate at any given time. The atrophy rate $\tilde{a}(\boldsymbol{x}, t)$ at any position $\boldsymbol{x}$ at time $t$ for a representative elementary volume of $V_{\boldsymbol{x} t}$ is defined as the negative rate of change of volume per unit volume: $\frac{\partial V_{\boldsymbol{x} t}}{\partial t}=-\tilde{a} V_{\boldsymbol{x} t}$.

Starting from initial configuration with zero displacement, for a small time $\Delta t$, using $\boldsymbol{v}=\boldsymbol{u} / \Delta t$, we have

$$
\nabla \cdot \boldsymbol{u}=-\tilde{a} \Delta t=-a
$$

where $\boldsymbol{u}$ is the displacement and $a$ is the amount of atrophy during the time $\Delta t$.

\subsection{Constrained Minimization of the Elastic Energy}

We do not explicitly model the neuronal loss and tissue remodeling at the microscopic level which requires biochemical and cellular physiological knowledge in detail. We abstract the phenomenon that evolves over several months or years in the brain. The model is based on the assumption that atrophy creates internal stress which results in the deformation minimizing a strain energy. Thus the brain parenchyma deforms with the prescribed atrophy by minimizing the strain energy.

Using Saint Venant-Kirchoff model for an elastic material, this can be expressed as the minimization of:

$$
R(\boldsymbol{u}, p)=\int \mu \operatorname{tr}\left(\boldsymbol{E}(\boldsymbol{u})^{2}\right)+\frac{\lambda}{2}(\operatorname{tr}(\boldsymbol{E}(\boldsymbol{u})))^{2}-\int p(\nabla \cdot \boldsymbol{u}+a)
$$

where $p$ is a Lagrange multiplier, $\mu$ and $\lambda$ are Lamé constants, and $\boldsymbol{E}$ is Langrangian Green strain defined as: $\boldsymbol{E}=\frac{1}{2}\left(\nabla \boldsymbol{u}+\nabla \boldsymbol{u}^{T}+\nabla \boldsymbol{u}^{T} \nabla \boldsymbol{u}\right)$. 
By taking a sufficiently small time step $\Delta t$, this deformation could be reasonably modelled as being of linear elastic in nature. For example, for a $2 \%$ global atrophy per year, we have $\Delta t=1$ year, and the atrophy during the year as $a=0.02$. This linear elastic assumption is done for a small time step only because remodelling occurs to eliminate (or at least attenuate) the internal stress after this deformation, thus leading to a creep flow. The partial attenuation only would lead to residual stress and plastic behavior.

Under linear elastic assumptions, minimizing the energy in equation (2) is equivalent to solving set of equations on the left in (3). In CSF region the mechanical behavior is different: the internal stress due to atrophy is not present. Thus we modify the equation on left of (3) to include the CSF region and get a combined equation as shown on the right. We set $(k=1, f=0)$ in CSF region and $(k=0, f=a)$ in gray and white matter (GM/WM) regions.

By imposing $k=1$ and $f=0$ in CSF, we remove the force term of the momentum equation and we allow CSF to expand as required at any place. This is reasonable because CSF gets produced every hour while the brain deformation due to atrophy takes place in months and years.

$$
\begin{aligned}
\mu \Delta \boldsymbol{u}-\nabla p & =(\mu+\lambda) \nabla a & \mu \Delta \boldsymbol{u}-\nabla p & =(\mu+\lambda) \nabla f \\
\nabla \cdot \boldsymbol{u} & =-a, & \nabla \cdot \boldsymbol{u}+k p & =-f,
\end{aligned}
$$

where $\Delta \boldsymbol{u}$ is a component-wise Laplacian of $\boldsymbol{u}$.

\section{Implemenation and Experimental Setup}

\subsection{Discretization with Finite Difference Method}

For the problem sizes typical in our application, direct solvers are impractical for solving equation (3). It needs a suitable combination of an iterative solver and a preconditioner to solve it. Furthermore, the pressure and velocity variables must be properly discretized to ensure stability. For discretization we use Finite Difference Method (FDM) with staggered grid. We chose FDM instead of FEM to avoid brain meshing and transporting computed variables from mesh to image at each iteration. This also allows us to solve the system in a grid that is of the same size as the input image where the grid fits naturally to the image. Tho solver is implemented with PETSc library [2], and the system is solved by distributed computing in the locally available cluster.

\subsection{Data Preprocessing}

The model requires to describe the atrophy in GM and WM of the brain at the level of MRI resolution. Thus we first need a segmentation of the desired regions. First the input image is skull stripped using ROBEX [7]. The computational domain for the solution of the PDE is obtained by finding the smallest cuboid region that completely encloses the skull stripped mask. In the current setting, 
we set the Dirchlet boundary of zero displacement on all the walls of this cuboid. For the regions lying on and outside the skull but inside the cuboid, we set $k=0$ and $f=0$ so that there is no volume loss or gain and no external force term to drive the deformation. The experiments show that there is no significant motion in the skull with this approach. The implementation will be extended in the future to impose Dirichlet boundary condition on the boundary of the skull itself by utilizing the skull stripped mask. We use FSL FAST [17] to segment the skull stripped image into GM, WM and CSF; and FSL FIRST [11] to segment hippocampi and amygdalae.

\subsection{Subject Specific Atrophy Map}

Skull stripping and tissue segmentation can be done directly in a subject specific MRI. This allows prescribing desired atrophy on the segmented regions of the subject MRI. For the regions that cannot be segmented directly on the subject MRI, if they are are available in atlases, we can propagate the required labels to the specific subject MRI by using non-rigid registration of the template atlas to the subject.

\section{Simulation Results and Discussion}

Investigation of differential brain atrophy dynamics in GM and WM: We used OASIS-30 atlas and the template brain image [10] to experiment with differential patterns of atrophy in GM and WM. Figure 1 shows displacement fields obtained when prescribing $2 \%$ global atrophy in two different ways. The left column shows the field when all of the atrophy is uniformly distributed in the GM only, while the middle column shows the field when all of the atrophy is uniformly distributed in the WM only. The right column shows the difference between the fields in these two cases i.e. GM-atrophy and WM-atrophy cases. We can see that the deformations are localized only on the cortical areas in the GM-atrophy case. In the WM-atrophy case, deformations are similar on the cortical surface but are much pronounced internally in WM areas and at the WM-GM interface.

Towards a Realistic Subject-Specific Model of AD: Figure 2 shows a simulation example for a subject brain MRI where $20 \%$ hippocampal atrophy (magnified hippocampal atrophy is prescribed here for illustrative purpose) is prescribed in addition to the uniform global $2 \%$ atrophy. One of the coronal slices is superimposed with the obtained deformation field on the left column. The right column shows the difference between the original image and the simulated image. The difference is mostly localized in the GM-WM interface and the CSFGM interface. The deformation field shows the expected large shrinkage of the hipoccampi that was prescribed. 


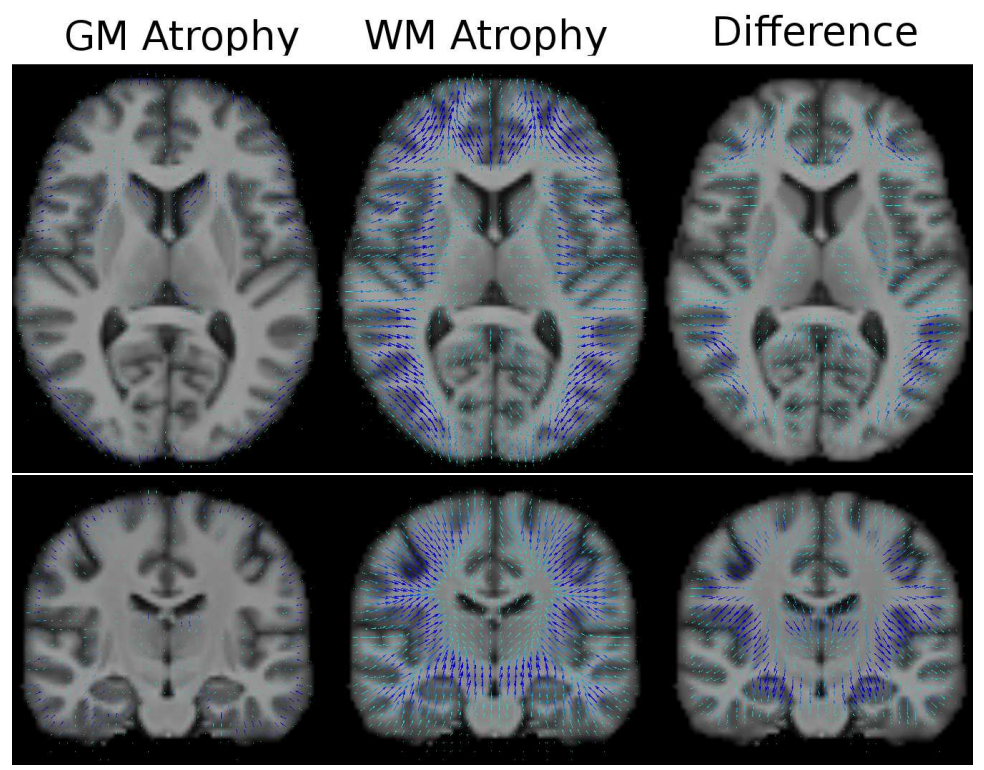

Fig. 1. Displacement fields superimposed over two views of the template MRI. Prescribed atrophy is the two percent of the total brain volume. Left: Atrophy only in the GM. Middle: Atrophy only in the WM. Right: Difference between displacement fields obtained in the first two cases.

These examples illustrate how we can use the proposed framework to explore the association of different atrophy patterns and their resulting deformation patterns with the image appearance in structural MRI.

The proposed model obtains deformation for one time step. The question remains on how we can further deform the brain by updating the atrophy map. This requires the knowledge of the evolution of atrophy over time. Here we take the simple approach of updating the atrophy map using the deformation field obtained in the first step and re-solving the system of equations (dissipating all the strain energy from the previous step).

\section{Conclusions}

We have proposed a new biologically plausible model of brain atrophy that provides a deformation with exactly the prescribed atrophy at any desired voxel of the image. The model is described by a PDE that is solved directly in the grid of the image resolution without the need of brain meshes. It allows to simulate anatomical changes of the brain based on specified atrophy patterns. We showed that this well-grounded forward model of the brain deformation in AD allows us to test hypotheses about the distribution of brain atrophy, and to study its impact on the observed changes in MR images. 


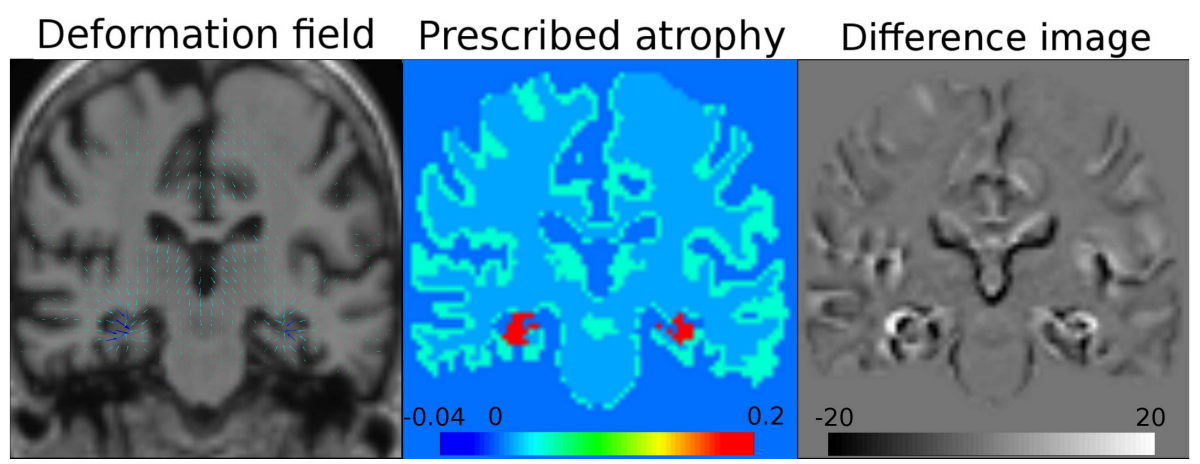

Fig. 2. Left: A coronal slice of a subject brain MRI superimposed with the obtained displacement field. Middle: Prescribed atrophy map of $2 \%$ global atrophy where GM and WM conribute $80 \%$ and $20 \%$ respectively and an additional $20 \%$ hippocampal atrophy. Right: Difference between the original and the simulated image.

We have provided a preliminary example towards the development of a realistic and biologically plausible subject-specific model of AD. Further developments of the present work will extend the personalization by including parameters concerning structural and biochemical information (from respectively diffusion weighted and PET imaging), and clinical/sociodemographical factors. The model opens a way to the development of novel instruments to be used in clinical trials for monitoring longitudinal brain changes with respect to simulated scenarios of pathological evolutions.

A biomarker serving as a ground truth for the model validation is challenging since there is no universally accepted biomarker for atrophy. One possible biomarker could be the pattern of accumulation of neurofibrillary tangles (NFTs) in the brain [3]. In future, imaging data based on novel tau aggregrating tracers [16] that provides the pattern of NFTs could be used as patient-specific prior for the initial atrophy map in the model, and then we could compare the outcome with the real images in order to establish the stage of the pathology.

Acknowledgements Part of this work was funded by the European Research Council through the ERC Advanced Grant MedYMA 2011-291080.

\section{References}

1. Ashburner, J., Ridgway, G.R.: Symmetric diffeomorphic modeling of longitudinal structural MRI. Frontiers in Neuroscience 6 (2012)

2. Balay, S., Brown, J., Buschelman, K., Gropp, W.D., Kaushik, D., Knepley, M.G., McInnes, L.C., Smith, B.F., Zhang, H.: PETSc Web page (2013), http://www.mcs.anl.gov/petsc

3. Braak, H., Braak, E.: Staging of Alzheimer's disease-related neurofibrillary changes. Neurobiology of Aging 16(3), 271-278 (1995) 
4. Camara, O., Scahill, R.I., Schnabel, J.A., Crum, W.R., Ridgway, G.R., Hill, D.L., Fox, N.C.: Accuracy assessment of global and local atrophy measurement techniques with realistic simulated longitudinal data. In: Ayache, N., Ourselin, S., Maeder, A. (eds.) MICCAI 2007, LNCS, vol. 4792, pp. 785-792. Springer, Heidelberg (2007)

5. Camara, O., Schweiger, M., Scahill, R.I., Crum, W.R., Sneller, B.I., Schnabel, J.A., Ridgway, G.R., Cash, D.M., Hill, D.L.G., Fox, N.C.: Phenomenological model of diffuse global and regional atrophy using finite-element methods. IEEE Transactions on Medical Imaging 25(11), 1417-30 (Nov 2006)

6. Frisoni, G.B., Fox, N.C., Jack, C.R., Scheltens, P., Thompson, P.M.: The clinical use of structural MRI in Alzheimer disease. Nature Reviews. Neurology 6(2), 67-77 (Feb 2010)

7. Iglesias, J.E., Liu, C., Thompson, P.M., Tu, Z.: Robust brain extraction across datasets and comparison with publicly available methods. IEEE Transactions on Medical Imaging 30(9), 1617-1634 (2011)

8. Johnson, R.T., Gibbs Jr, C.J.: Creutzfeldt-Jakob disease and related transmissible spongiform encephalopathies. New England Journal of Medicine 339(27), 19942004 (1998)

9. Karaçali, B., Davatzikos, C.: Simulation of tissue atrophy using a topology preserving transformation model. IEEE Transactions on Medical Imaging 25(5), 649-52 (May 2006)

10. Klein, A., Tourville, J., et al.: 101 labeled brain images and a consistent human cortical labeling protocol. Frontiers in Neuroscience 6, 171-171 (2011)

11. Patenaude, B., Smith, S.M., Kennedy, D.N., Jenkinson, M.: A bayesian model of shape and appearance for subcortical brain segmentation. Neuroimage 56(3), 907-922 (2011)

12. Pieperhoff, P., Südmeyer, M., Hömke, L., Zilles, K., Schnitzler, A., Amunts, K.: Detection of structural changes of the human brain in longitudinally acquired MR images by deformation field morphometry: methodological analysis, validation and application. NeuroImage 43(2), 269-87 (Nov 2008)

13. Sharma, S., Noblet, V., Rousseau, F., Heitz, F., Rumbach, L., Armspach, J.: Evaluation of brain atrophy estimation algorithms using simulated ground-truth data. Medical Image Analysis 14(3), 373-89 (Jun 2010)

14. Sharma, S., Rousseau, F., Heitz, F., Rumbach, L., Armspach, J.: On the estimation and correction of bias in local atrophy estimations using example atrophy simulations. Computerized Medical Imaging and Graphics 37(78), 538 - 551 (2013)

15. Smith, A.D.C., Crum, W.R., Hill, D.L., Thacker, N.A., Bromiley, P.A.: Biomechanical simulation of atrophy in MR images. In: Medical Imaging 2003. pp. 481-490. International Society for Optics and Photonics (2003)

16. Zhang, W., Arteaga, J., Cashion, D., et al: A highly selective and specific PET tracer for imaging of tau pathologies. Journal of Alzheimer's Disease 31(3), 601-612 (2012)

17. Zhang, Y., Brady, M., Smith, S.: Segmentation of brain MR images through a hidden markov random field model and the expectation-maximization algorithm. IEEE Transactions on Medical Imaging 20(1), 45-57 (2001) 